\title{
Large cavernous carotid artery aneurysm with spontaneous thrombosis: is there more to a change in morphology than there seems to be? Illustrative case
}

\author{
Yoriko Kato, MD, ${ }^{1,3}$ Wataro Tsuruta, MD, PhD, ${ }^{1}$ Hisayuki Hosoo, MD, $\mathrm{PhD},{ }^{2}$ and Tetsuya Yamamoto, MD, $\mathrm{PhD}{ }^{3}$ \\ ${ }^{1}$ Department of Endovascular Neurosurgery, Toranomon Hospital, Tokyo, Japan; ${ }^{2}$ Department of Neurosurgery, Faculty of Medicine, University of Tsukuba, Ibaraki, Japan; and \\ ${ }^{3}$ Department of Neurosurgery, School of Medicine, Yokohama City University, Kanagawa, Japan
}

\begin{abstract}
BACKGROUND The pathogenesis and endovascular treatment strategy for spontaneously thrombosed unruptured cerebral aneurysms have not yet been comprehensively described.

OBSERVATIONS The authors reported on a 78-year-old woman who had large bilateral unruptured cavernous carotid artery aneurysms that induced chronic disseminated intravascular coagulation and acquired factor XIII deficiency. The right aneurysm was symptomatic and partially thrombosed. Hemorrhagic diathesis and abnormal values of laboratory data improved after administration of recombinant human thrombomodulin followed by endovascular treatment in which three pipeline embolization devices were deployed for the right aneurysm.

LESSONS To the best of the authors' knowledge, this was the first report of an unruptured cerebral aneurysm leading to coagulation disorders with clinical manifestation that was treated successfully by endovascular intervention after intensive perioperative management.
\end{abstract}

https://thejns.org/doi/abs/10.3171/CASE21288

KEYWORDS disseminated intravascular coagulation; rheumatoid arthritis; spontaneous thrombosis; unruptured cerebral aneurysm

The intraaneurysmal thrombosis of large cerebral aneurysms is not uncommon, with the frequency of spontaneous thrombosis reported to be $13 \%$ to $60 \%$. $^{1-6}$ These reports are retrospective cohort studies that include patients with subarachnoid hemorrhage. However, few reports to date describe pathogenesis and suggest treatment strategies for spontaneously thrombosed unruptured cerebral aneurysms. ${ }^{4,7,8}$ Endovascular treatment, including the deployment of a flow diverter stent, sometimes requires device delivery distally through the aneurysm, and intraaneurysmal thrombus presents a risk of intraoperative embolism. In addition, it has been reported that the rate of complete occlusion after the placement of a flow diverter stent is low in partially thrombosed intracranial aneurysms. ${ }^{9}$ To achieve successful endovascular treatment, including perioperative management, it is important to understand the pathophysiology of thrombosed unruptured cerebral aneurysms. We report a case of large cavernous carotid artery (CCA) aneurysms with spontaneous thromboses presenting enhanced fibrinolytic type of chronic disseminated intravascular coagulation (DIC) and acquired factor XIII (FXIII) deficiency associated with thrombus formation. When DIC treatment with recombinant human thrombomodulin (rhTM) that inhibits functions of thrombin was performed, the thrombus in the aneurysm dissolved and shrank. The mechanism of unruptured cerebral aneurysm thrombosis will be considered from the laboratory findings and treatment course of this case.

\section{Illustrative Case}

The patient was a 78-year-old woman. She had experienced right ptosis that gradually deteriorated over 5 months and then visited her previous physician because of diplopia. Magnetic resonance imaging (MRI) showed large bilateral CCA aneurysms. She

ABBREVIATIONS AAA = abdominal aortic aneurysm; CCA = cavernous carotid artery; DIC = disseminated intravascular coagulation; FDP = fibrin degradation product; FXIII = factor XIII; ILT = intraluminal thrombus; MRI = magnetic resonance imaging; NF = nuclear factor; OPG = osteoprotegerin; PED = pipeline embolization device; $\mathrm{RA}=$ rheumatoid arthritis; $\mathrm{RANK}=$ receptor activator of NF $\kappa \beta ;$ RANKL = RANK ligand; $r \mathrm{hTM}=$ recombinant human thrombomodulin; TAT = thrombin antithrombin complex; TNF = tumor necrosis factor.

INCLUDE WHEN CITING Published August 16, 2021; DOI: 10.3171/CASE21288.

SUBMITTED May 8, 2021. ACCEPTED July 2, 2021.

(C) 2021 The authors, CC BY-NC-ND 4.0 (http://creativecommons.org/licenses/by-nc-nd/4.0/). 
was diagnosed with rheumatoid arthritis (RA) when she was 35 years old and was taking salazosulfapyridine. Deformity and swelling of joints were observed in her hands, and her simplified disease activity index score was 5.1 , indicating a low disease activity state in RA on admission. She had experienced a cerebral infarction in her left parietal lobe and was taking $100 \mathrm{mg}$ of aspirin per day.

Angiography revealed a partially thrombosed right CCA aneurysm measuring $23 \times 19 \mathrm{~mm}$ (Fig. 1). During the angiography, she experienced a massive groin hematoma at the puncture site of the 4-Fr sheath without any procedural cause. Some laboratory parameters of coagulation and fibrinolysis registered in the abnormal range, such as D-dimer, which was significantly high. Systematic examinations conducted by a hematologist revealed enhanced fibrinolytic type of chronic DIC and acquired FXIII deficiency induced by the thrombosed intracranial aneurysm (Table 1). The patient was treated with rhTM and fresh frozen plasma. Three weeks after the initial angiography, FXIII activity had improved, and the endovascular treatment using three pipeline embolization devices (PEDs; Medtronic) was performed on the symptomatic right CCA aneurysm. The thrombus in the aneurysm was significantly reduced, and the characteristics of the aneurysm had unexpectedly changed over the 3 weeks during which we had been treating the DIC and acquired FXIII deficiency. The patient's right ptosis and diplopia started to improve 2 days after the endovascular treatment, and clinical complications related to hemorrhagic disorder were not seen again. A 1-year follow-up angiogram showed that the aneurysm was slightly filled, indicating grade B on the O'Kelly-Marotta scale. The mass effect had been released, and the patient recovered completely from the right ptosis and ophthalmoplegia.

\section{Discussion}

\section{Observations}

Intraaneurysmal thrombus in a cerebral aneurysm is believed to be initially triggered by endothelial damage caused by hemodynamic stress. ${ }^{10}$ In the case of CCA aneurysm, slow flow induced by an anatomical distortion of the parent artery and an increase in volume-to-neck ratio also have been suggested as possible anatomical factors that induce thrombosis. ${ }^{1,6}$

The mechanism of partially thrombosed aneurysms is believed to be the formation of subadventitial hematoma generated by a cascade of leukocytes released from the vasa vasorum into the adventitia; that is, the process is mediated from the outside of the vessel. It has been argued that the main mechanism of cerebral aneurysm growth is intramural hematoma of the aneurysm caused by rupture of the adventitia, often found in arterial dissection, and is not due to intraluminal thrombus (ILT). ${ }^{10}$

Among recent reports investigating the wall microstructure of thrombosed cerebral aneurysms using high spatial resolution MRI techniques, high-field 7T contrast-enhanced MRI was directly contrasted with pathological findings in 14 thrombosed cerebral aneurysms. Inner wall enhancement was seen in all aneurysms, indicating neovascularization of the intima adjacent to the ILT. The outer wall enhancement observed in 7 cases correlated to the formation of vasa vasorum in the outer layer of the aneurysm. ${ }^{11}$ Thrombosed cerebral aneurysms are considered to have two different mechanisms and structures to form ILT and intramural hematoma.

The intraaneurysmal thrombus that induced systemic coagulation/fibrinolytic abnormalities in the present case is considered an
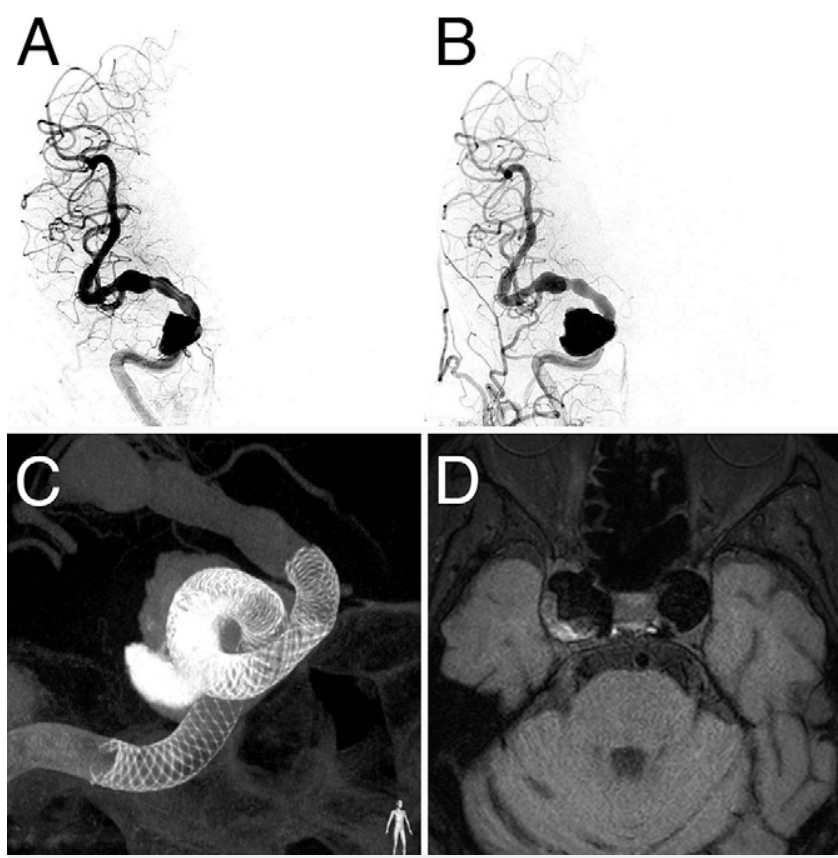

FIG. 1. A: Initial angiography in anteroposterior view shows the right CCA aneurysm with spontaneous thrombosis. B: Angiography in anteroposterior view of the right aneurysm during the endovascular treatment performed 3 weeks after the initial angiography. C: Right internal carotid artery with three PEDs; working angle. D: 3T MRI improved motion sensitized driven equilibrium black blood imaging shows CCA aneurysms and thrombus in the right aneurysm.

ILT, and its mechanism and structure were different from the previously discussed layered intramural hematoma within the adventitia.

Abdominal aortic aneurysm (AAA) is now considered to be an atherothrombotic disease characterized by an ILT rather than arteriosclerosis. The constant exposure of the subendothelial tissue factor to circulation induces the formation of a prothrombinase complex consisting of FXa and FVa, and FXIIla crosslinks the fibrin monomers, leading to fibrin polymerization. The primary hemostatic thrombus is stabilized, and then a secondary platelet-fibrin thrombus is formed. This is the initial stage of the ILT, and it leads to increase in aortic diameter and loss of normal vessel structure. The ILT also acts a source of inflammatory cytokines and fibrinolytic products. ${ }^{12}$ Recent meta-analysis has reported an increase in Ddimer and thrombin antithrombin complex (TAT) in AAA cases, and an increase in D-dimer is positively correlated with AAA growth. ${ }^{13}$ Since the report by Fine et al. in 1967 showing that AAA induces DIC, the incidence of AAA with symptomatic DIC caused by excess consumption of coagulation factors has been reported as $0.5 \%$ to $4 \%$, and perioperative evaluation of hemostatic derangement should be considered, even if the patient with AAA has no clinical manifestation of hemorrhagic diathesis. ${ }^{14,15}$

In typical cases of DIC, those caused by sepsis, inflammatory cytokines inhibit the expression of TM, an anticlotting protein in the vascular endothelium, thereby activating intravascular coagulation. In most cases, we would expect fibrinolysis to be activated as the body attempts to dissolve the multiple microthrombi produced by such abnormally activated coagulation. Asakura reported that cytokines also cause 
TABLE 1. Laboratory data on DIC and RA

\begin{tabular}{|c|c|c|c|c|c|}
\hline Variable & Reference Range* & 3 Wks Before Endovascular Treatment & 3 Days Before & 1 Wk After & 1 Yr After \\
\hline D-dimer $(\mu \mathrm{g} / \mathrm{mL})$ & $<1$ & 51.0 & 17.6 & 20.7 & 31.8 \\
\hline PTINR & & 1.02 & 1.04 & 1.08 & 1.07 \\
\hline APTT & $27.0-40.0$ & 30.0 & 29.7 & 32.6 & 29.8 \\
\hline Fibrinogen (mg/dL) & $150-300$ & 144.5 & 273.5 & 283.8 & 323.5 \\
\hline FDP $(\mu \mathrm{g} / \mathrm{mL})$ & $<5$ & 95.9 & 23.8 & 29.9 & 48.6 \\
\hline Platelet $(\times 1,000 / \mu \mathrm{L})$ & $155-350$ & 104 & 174 & 148 & 162 \\
\hline FXIII activity (\%) & $70-140$ & 40 & 87 & 73 & \\
\hline vWF activity (\%) & $50-150$ & 209 & & 129 & \\
\hline TAT $(\mu \mathrm{g} / \mathrm{L})$ & $<4.0$ & 37.5 & & 16.3 & 9.0 \\
\hline $\mathrm{PIC}(\mu \mathrm{g} / \mathrm{mL})$ & $<0.8$ & 5.2 & & 1.2 & 1.6 \\
\hline Rheumatoid factor $(\mathrm{U} / \mathrm{mL})$ & $0-15$ & 57 & & 56 & \\
\hline CRP (mg/dL) & $0.0-0.3$ & 0.1 & 0.3 & 1.0 & 0.5 \\
\hline FDP/D-dimer ratio & & 1.9 & 1.4 & 1.4 & 1.5 \\
\hline
\end{tabular}

APTT = activated partial thromboplastin time; $\mathrm{CRP}=\mathrm{C}$-reactive protein; $\mathrm{PIC}=$ plasmin inhibitor complex; PTINR = prothrombin time international normalized ratio; $\mathrm{VWF}=$ von Willebrand factor.

* The ranges used at the hospital are for adults.

the plasminogen activator inhibitor to be overexpressed, which then suppresses fibrinolysis, and DIC caused by sepsis results in suppressed fibrinolytic-type DIC. On the other hand, in cases of DIC caused by AAA, fibrinolysis is highly activated in response to coagulation activation, resulting in enhanced fibrinolytic-type DIC. Therefore, sepsis-induced DIC results in suppressed fibrinolytic-type DIC, whereas AAA-induced DIC results in enhanced fibrinolytic-type DIC. Hemostatic plugs tend to be easily dissolved by strongly activated fibrinolysis; a bleeding disorder could become severe. ${ }^{16}$ During angiography, the patient manifested bleeding diathesis for the first time in the form of groin hematoma. We consider that excessive consumption of coagulation factors caused by enhanced fibrinolytic type of chronic DIC led to deficiency of FXIII, also known as a fibrin stabilizing factor, which then induced the bleeding symptom.

TM suppresses the coagulant activity of thrombin and mediates antiinflammatory activities by enhancing the activation of protein $\mathrm{C}$, thereby playing an important role in inflammatory protection during AAA formation and suppressing AAA growth. ${ }^{12,17}$ In this case, administering rhTM as a treatment for chronic DIC is considered to have contributed to the reduction of thrombus in the aneurysm. RhTM-responsive thrombolysis suggests intraaneurysmal thrombosis crosstalk with a systemic coagulation/fibrinolysis cascade.

In the laboratory data of the case reported here, FXIII was consumed to stabilize the thrombus, followed by a decrease in the level of fibrinogen, an increase in the level of fibrin degradation product (FDP), and an elevation in both TAT and plasmin inhibitor complex, indicating enhanced fibrinolytic-type DIC. ${ }^{16}$ Systemic elaborate examinations concluded there was no other disease that could cause chronic DIC except the large thrombosed CCA aneurysm. After the PED treatment, the fibrinogen and platelet levels returned to normal, FDP decreased, and no bleeding diathesis was observed clinically. Over the whole clinical course with hematological evaluations and the endovascular treatment, we consider that the intraaneurysmal thrombus that was constantly exposed to cerebral blood flow acted as a biologically active ILT and induced chronic DIC, causing acquired FXIII deficiency.

The markedly elevated level of D-dimer decreased once after the PED placement but remained high, which is considered to be related to the patient's 40-year chronic RA condition.

Nuclear factor (NF) $\kappa \beta$ activation is the main inflammatory condition of RA. Zhang et al. reported that a hypercoagulation state caused by abnormal activation of NF $\kappa \beta$ is a major clinical background to RA, and D-dimer is significantly higher in patients with RA than healthy controls. ${ }^{18}$

Recently, an alternative NF $\kappa \beta$ activation pathway triggered by activation of the tumor necrosis factor (TNF) receptor superfamily has been reported. ${ }^{19}$ Receptor activator of NF $\kappa \beta$ (RANK), a transmembrane protein belonging to the TNF receptor superfamily, binds to its ligand (RANKL) and triggers the NF $\kappa \beta$ activation pathway. Osteoprotegerin (OPG), which also belongs to the TNF receptor superfamily, controls this RANKL/RANK interaction. ${ }^{20,21}$ In recent years, OPG not only has been identified as a regulator in bone metabolism but also is expressed in vascular smooth muscle cells and endothelial cells, and it has emerged as a protective modulator in cardiovascular disease. ${ }^{22}$

The increased risk of cardiovascular disease in patients with RA is well known. ${ }^{23-25}$ Compared to healthy controls, the expression of the OPG gene and OPG serum level are significantly increased in patients with RA, and the serum OPG concentrations are increased in patients with RA who have a history of cerebrovascular disease compared to those who do not. Therefore, the OPG/RANKL/RANK axis plays an important role in accelerating atherosclerotic alteration and cardiovascular risk. ${ }^{26,27}$

It has been reported that blood OPG concentrations are higher in patients with $\mathrm{AAA}$ and are positively correlated with AAA progression and that ILT itself can be a source of OPG release. OPG helps reduce cardiovascular disease risk; at the same time, OPG concentrations increase with atherosclerotic lesions. ${ }^{28,29}$ Measuring OPG 
concentrations in the present case would have helped clarify the relation between RA and cerebral aneurysm with ILT.

So far, there have been no reports of unruptured cerebral aneurysms inducing acquired FXIII deficiency or DIC.

\section{Lessons}

When a large unruptured cerebral aneurysm with thrombus is observed, it should be examined not only from the perspective of local characteristics of the aneurysm caused by anatomical factors but also in terms of potential relations with systemic coagulation/ fibrinolytic cascade and underlying chronic inflammatory disease. The spontaneously thrombosed intracranial aneurysm might crosstalk with the systemic coagulation/fibrinolytic function. Understanding these relations will help develop the most effective treatment strategy and perioperative management.

\section{Acknowledgments}

We would like to thank the patient who kindly provided consent allowing the publication of details of this case. Dr. Shinsuke Takagi, a board-certified hematologist, treated the patient, generously reviewed the manuscript, and provided thoughtful comments.

\section{References}

1. Das KK, Singh G, Pandey S, Bhaisora KS, Jaiswal A, Behari S. Completely thrombosed giant intracranial aneurysm with spontaneous thrombosis of the parent artery: is it nature's divine intervention and a self-cure? World Neurosurg. 2018;118:132-138.

2. Güresir E, Wispel C, Borger V, Hadjiathanasiou A, Vatter H, Schuss $P$. Treatment of partially thrombosed intracranial aneurysms: singlecenter series and systematic review. World Neurosurg. 2018;118:e834-e841.

3. Yang K, Park JC, Ahn JS, Kwon DH, Kwun BD, Kim CJ. Characteristics and outcomes of varied treatment modalities for partially thrombosed intracranial aneurysms: a review of 35 cases. Acta Neurochir (Wien). 2014;156(9):1669-1675.

4. Roccatagliata L, Guédin P, Condette-Auliac S, et al. Partially thrombosed intracranial aneurysms: symptoms, evolution, and therapeutic management. Acta Neurochir (Wien). 2010;152(12):2133-2142.

5. Whittle IR, Williams DB, Halmagyi GM, Besser M. Spontaneous thrombosis of a giant intracranial aneurysm and ipsilateral internal carotid artery. Case report. J Neurosurg. 1982;56(2):287-289.

6. Whittle IR, Dorsch NW, Besser M. Spontaneous thrombosis in giant intracranial aneurysms. J Neurol Neurosurg Psychiatry. 1982;45(11):1040-1047.

7. Kooshkabadi A, Jankowitz B, Choi PA, Weiner GM, Greene S. Thrombosis and spontaneous recanalization of a giant intracranial aneurysm: diagnostic and management pearls in a pediatric patient. J Neurosurg Pediatr. 2015;15(1):78-81.

8. Atkinson JL, Lane JI, Colbassani HJ, Llewellyn DM. Spontaneous thrombosis of posterior cerebral artery aneurysm with angiographic reappearance. Case report. J Neurosurg. 1993;79(3):434-437.

9. Foreman PM, Salem MM, Griessenauer CJ, et al. Flow diversion for treatment of partially thrombosed aneurysms: a multicenter cohort. World Neurosurg. 2020;135:e164-e173.

10. Krings T, Piske RL, Lasjaunias PL. Intracranial arterial aneurysm vasculopathies: targeting the outer vessel wall. Neuroradiology. 2005;47(12):931-937.

11. Sato T, Matsushige T, Chen B, et al. Wall contrast enhancement of thrombosed intracranial aneurysms at 7T MRI. AJNR Am J Neuroradiol. 2019;40(7):1106-1111.

12. Cameron SJ, Russell HM, Owens AP 3rd. Antithrombotic therapy in abdominal aortic aneurysm: beneficial or detrimental? Blood. 2018;132(25):2619-2628.
13. Davies RS, Abdelhamid M, Wall ML, Vohra RK, Bradbury AW, Adam DJ. Coagulation, fibrinolysis, and platelet activation in patients undergoing open and endovascular repair of abdominal aortic aneurysm. J Vasc Surg. 2011;54(3):865-878.

14. Fine NL, Applebaum J, Elguezabal A, Castleman L. Multiple coagulation defects in association with dissecting aneurysm. Arch Intern Med. 1967:119(5):522-526.

15. Koba S, Yamaguchi T, Miki K, Makihara H, Imashuku S. Management of chronic disseminated intravascular coagulation associated with aortic aneurysm/dissection. Case Rep Hematol. 2019;2019:6204652.

16. Asakura $\mathrm{H}$. Classifying types of disseminated intravascular coagulation: clinical and animal models. J Intensive Care. 2014;2(1):20

17. Lai CH, Shi GY, Lee FT, et al. Recombinant human thrombomodulin suppresses experimental abdominal aortic aneurysms induced by calcium chloride in mice. Ann Surg. 2013;258(6):1103-1110.

18. Zhang P, Liu J, Tan B. Hypercoagulation in patients with rheumatoid arthritis correlates with activation of Act1/NF-Kb signaling pathway. J Rheum Dis Treat. 2015;1:24.

19. Noort AR, Tak PP, Tas SW. Non-canonical NF-кB signaling in rheumatoid arthritis: Dr Jekyll and Mr Hyde? Arthritis Res Ther. 2015:17(1):15.

20. Schett G, Redlich K, Smolen JS. The role of osteoprotegerin in arthritis. Arthritis Res Ther. 2003;5(5):239-245.

21. Remuzgo-Martínez S, Genre F, López-Mejías R, et al. Expression of osteoprotegerin and its ligands, RANKL and TRAIL, in rheumatoid arthritis. Sci Rep. 2016;6:29713.

22. Miyata T, Minami M, Kataoka $\mathrm{H}$, et al. Osteoprotegerin prevents intracranial aneurysm progression by promoting collagen biosynthesis and vascular smooth muscle cell proliferation. J Am Heart Assoc. 2020;9(17):e015731.

23. Shovman O, Tiosano S, Comaneshter D, Cohen AD, Amital H, Sherf M. Aortic aneurysm associated with rheumatoid arthritis: a population-based cross-sectional study. Clin Rheumatol. 2016;35(11):2657-2661.

24. Pujades-Rodriguez M, Duyx B, Thomas SL, et al. Rheumatoid arthritis and incidence of twelve initial presentations of cardiovascular disease: a population record-linkage cohort study in England. PLoS One. 2016;11(3):e0151245.

25. Ahlers MJ, Lowery BD, Farber-Eger E, et al. Heart failure risk associated with rheumatoid arthritis-related chronic inflammation. J Am Heart Assoc. 2020;9(10):e014661.

26. Dessein PH, López-Mejias R, González-Juanatey C, et al. Independent relationship of osteoprotegerin concentrations with endothelial activation and carotid atherosclerosis in patients with severe rheumatoid arthritis. J Rheumatol. 2014;41(3):429-436.

27. López-Mejias R, Ubilla B, Genre F, et al. Osteoprotegerin concentrations relate independently to established cardiovascular disease in rheumatoid arthritis. J Rheumatol. 2015;42(1):39-45.

28. Koole D, Hurks R, Schoneveld A, et al. Osteoprotegerin is associated with aneurysm diameter and proteolysis in abdominal aortic aneurysm disease. Arterioscler Thromb Vasc Biol. 2012;32(6):1497-1504.

29. Vorkapic E, Kunath A, Wågsäter D. Effects of osteoprotegerin/ TNFRSF11B in two models of abdominal aortic aneurysms. Mol Med Rep. 2018;18(1):41-48.

\section{Disclosures}

The authors report no conflict of interest concerning the materials or methods used in this study or the findings specified in this paper.

\section{Author Contributions}

Conception and design: Kato, Tsuruta, Hosoo. Acquisition of data: Kato, Tsuruta, Hosoo. Analysis and interpretation of data: Kato, Hosoo. 
Drafting the article: Kato. Critically revising the article: Kato, Hosoo, Yamamoto. Reviewed submitted version of manuscript: all authors. Approved the final version of the manuscript on behalf of all authors: Kato. Administrative/technical/material support: Kato, Hosoo. Study supervision: Kato, Tsuruta.

\section{Correspondence}

Yoriko Kato: Toranomon Hospital, Tokyo, Japan. yoriko@pc4.so-net.ne.jp. 